\title{
Site-specific mutagenesis in cells with normal DNA repair systems: transitions produced from DNA carrying a single $\mathrm{O}^{6}$-alkylguanine
}

\author{
Robert W.Chambers \\ Department of Biochemistry, Dalhousie University, Halifax, Nova Scotia B3H 4H7, Canada
}

Received August 14, 1990; Revised and Accepted April 5, 1991

\begin{abstract}
This paper describes a systematic study of transition frequencies produced in vivo when a homologous series of $0^{6}$-alkylguanine residues located at a preselected position in gene G of $\phi X 174$ form I' DNA (double-stranded, circular, covalently-closed, relaxed) is transfected into spheroplasts from two strains of Escherichia coli having normal DNA repair systems. Mutant frequencies were measured as percent of total phage produced by single bursts. The results are: (A) Synthetic DNA without any alkyl group gave a transition frequency of $0.02 \%$. (B) In $E$. coli $A B 1157$, the frequencies fall into two groups depending on the alkyl group: methyl and ethyl, $8-11 \%$; $n$-propyl and $n$-butyl $\sim 0.9 \%$. (C) The average transition frequencies were higher in AB1157 than in $\mathbf{6 6 0 0}$. These data demonstrate that a single $0^{6}$-alkylguanine residue can produce a specific transition at significant frequencies in cells with normal repair systems and that the mutant frequency depends upon the nature of the alkyl group and the cell type.
\end{abstract}

\section{INTRODUCTION}

The experiments reported in this paper were designed with 4 objectives in mind: (a) to re-examine our earlier results, which, among other things, showed that a single $\mathrm{O}^{6}$-methylguanine residue (MeG) in $\phi X 174$ form $I^{\prime}$ DNA produces a site-specific transition at high frequency in cells with normal DNA repair systems (1); (b) to extend our early results by examining the mutagenic activity of $\mathrm{O}^{6}$-methyl-, ethyl-, $n$-propyl- and $n$ butylguanine residues in DNA; (c) to compare the mutant frequencies produced from these $\mathrm{O}^{6}$-alkylguanines in two closely related cell lines, $E$. coli $\mathrm{AB} 1157$ and $\mathrm{C} 600$, both of which are derived from $E$. coli $\mathrm{K} 12$ and are believed to have normal DNA repair systems; and (d) to compare our results obtained with $\mathrm{O}^{6}$-methylguanine with those reported by other laboratories $(2,3,4)$

\section{MATERIALS AND METHODS}

Bacterial strains

E. coli $\mathrm{C} 600 \mathrm{~F}^{-} \phi \mathrm{X}^{\mathrm{R}}$ thi-1 leu-6 lacY1 tonA21 supE44 $\lambda^{-}$was obtained from Dr. Sunil Palchaudary; its resistance to $\phi X 174$ was checked; its suppressor activity was verified by transfection of spheroplasts with DNA isolated from $\phi X$ Gam3, a $\phi X 174$ mutant carrying an amber mutation in the third codon of gene G (5).

$\mathrm{AB} 1157 \mathrm{~F}^{-} \phi-\mathrm{X}^{\mathrm{R}}$ thr-1 ara-14 leu-B6s (gpt-proA)lacY1 tsx-33 thi-1 supE44 galK2 $\lambda^{-}$his-G4 rpsL31 xyl-5 mtl-1 argE3 thi-1 was obtained from Dr. Barbara Backman at the $E$. coli Genetic Stock Center, Department of Microbiology, Yale University, School of Medicine New Haven, Connecticut; all the amino acid and sugar markers were verified; the suppressor activity was demonstrated with $\phi \mathrm{XGam3}$ DNA.

$\mathrm{HF} 4738 \mathrm{Su}^{+} \phi \mathrm{X}^{\mathrm{S}}$ recA, a K12/C hybrid, was obtained from the late Dr. Paul Howard-Flanders; it was shown to have the same suppression pattern as HF4714, which inserts Gln at amber codons $(5,6)$; its sensitivity to ultraviolet light $(254 \mathrm{~nm})$ was demonstrated by a quantitative survival curve compared to $\mathrm{AB} 1157$ at various exposures.

$E$. coli $\mathrm{C} \phi \mathrm{X}^{\mathrm{S}}$ was obtained from the American Type Culture Collection. It is a wild type host for bacteriophage $\phi \mathrm{X} 174$.

Other materials

The oligonucleotides used as primers for DNA synthesis were prepared as described previously (7). Wild-type bacteriophage $\phi X 174$ was grown by the inhibited lysis procedure exactly as described by Geschwinder and Hofschneider (8); the virus was purified by isopycnic centrifugation followed by gel filtration through Sephacryl 400 (Pharmacia) before extracting the DNA in the usual manner. Heteroduplex DNA carrying $\mathrm{O}^{6}$-alkylguanine at position 2401 of the minus strand was synthesized and purified as before (1). Spheroplasts were prepared from $E$. coli $\mathrm{AB} 1157$ and $\mathrm{C} 600$ as described by Malling et al. (9); only those preparations giving $>90 \%$ spheroplasts and good transfection efficiencies when assayed with wt phage were used.

\section{Measurement of Transition Frequencies}

Transfection was carried out as described by Guthrie and Sinsheimer except that incubation was at $37^{\circ}(10)$; the concentrations of DNA and spheroplasts were equal $\left(5 \times 10^{8} / \mathrm{ml}\right)$. All four modified DNAs, along with unmodified (synthetic) DNA and viral DNA, were assayed simultaneously. Lysis was effected by shaking the reaction mixture with $0.1 \mathrm{ml}$ of chloroform. The lysates were filtered through a sterile Millipore membrane $(0.45 \mu \mathrm{m})$ to removed debris and unlysed 
cells. Suitably diluted aliquots were titered on $E$. coli HF4738 using $R$ soft agar and $R$ plates (11). Using titers as a guide, the lysates were diluted so that a suitable aliquot gave about 100 pfu/plate. Three aliquots were plated on $E$. coli HF4738. All the plaques on each plate were gridded onto prepoured lawns of $\mathrm{HF} 4738$ and $E$. coli $\mathrm{C}$. The plates were incubated at $37^{\circ}$ for $5 \mathrm{~h}$ and compared. Wild type phage gives plaques on both lawns; the amber mutant, Gam3 (specific transition), gives plaques only on HF4738.

The mutant frequencies are expressed as percent of the phage examined (200-300) from each transfection. Three transfections were carried out and analyzed for each modified DNA with each of the cell types (AB1157 and C600). Where noted different batches of spheroplasts were also examined. The mean mutant frequency and the standard deviation were calculated. Since each transfection was carried out simultaneously with all the synthetic DNAs, the results are directly comparable. The statistical significance of the results were calculated from the test statistic: $t^{\prime}=\left(y_{1}-y_{2}\right) / \sqrt{ }\left\{\left[\left(s_{1}\right)^{2} / n_{1}\right]+\left[\left(s_{2}\right)^{2} / n_{2}\right]\right\}$ where $y_{1}$ and $y_{2}=$ the mean mutant frequencies for different alkyl groups or for the same alkyl group in different host cells; $s_{1}$ and $s_{2}=$ the standard deviations; $n_{1}$ and $n_{2}=$ the number of transfections (12).

\section{RESULTS}

The experimental design is outlined in Figure 1. The details are given in Materials and Methods. The cells used for transfection are at least $90 \%$ spheroplasts (by actual microscopic count). In general, these spheroplasts do not lyse spontaneously during incubation. Although it is possible that an occasional cell does lyse prematurely, the virus released cannot infect the intact bacilli that are invariably present in the spheroplast preparations because both $E$. coli $\mathrm{AB} 1157$ and $\mathrm{C} 600$ are resistant to the phage. Therefore, these are 'single burst' experiments.

Very low frequencies can be detected with the 'gridding assay' described in Methods and Materials. In order to rule out the possibility that the assay picks up amber mutants generated spontaneously at either the selected site or some other, I examined phage production from synthetic DNA made in exactly the same way and at the same time as the modified DNAs. Only one mutant was found among 4,424 plaques gridded in various experiments;

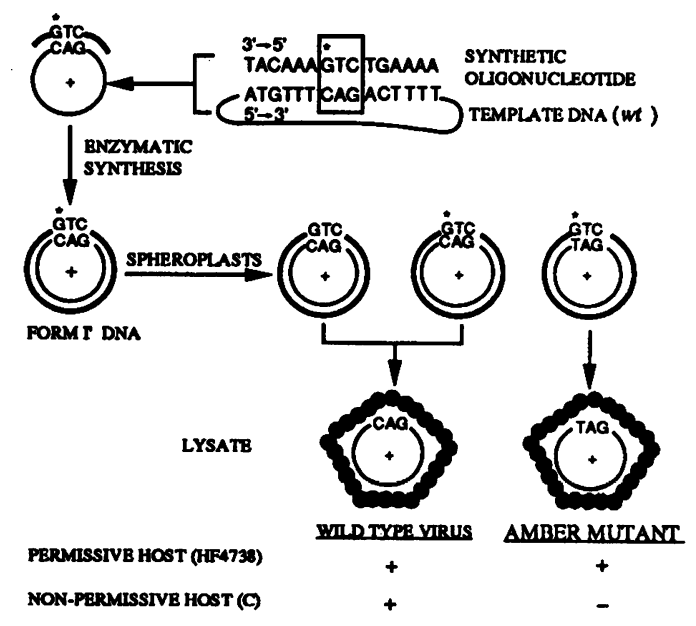

Figure 1. Experimental design. Alkyl group, *. thus, the mutation frequency for the control DNA is about $0.02 \%$. The specificity of the phenotypic screen and the results with control DNA indicate that sequencing to confirm the amber mutation is unnecessary. However, when sequencing has been done, the expected mutation was found in each case. Other kinds of mutations that would not be picked up by the assay are possible, but these occur at low frequency compared to the transition (unpublished data) and are irrelevant to this particular study.

The data obtained from 30 separate transfections with modified DNAs are shown in Table 1. The data are given as mutant frequencies, which is a measure of the number of mutants present in samples drawn from the total phage population. The data are normalized by calculating the ratio of mutants to total phage. When the data is expressed this way differences in total phage production do not affect the mutant frequency. For example, three transfections of $E$. coli $\mathrm{C} 600$ spheroplasts with DNA carrying MeG gave the following total phage (T) and mutant frequencies (M): (a) $\mathrm{T}=1,100, \mathrm{M}=2.8 \%$; (b) $\mathrm{T}=7,600, \mathrm{M}=2.8 \%$; (c) $\mathrm{T}=4,800, \mathrm{M}=2.1 \%$. Thus, for a 7-fold variation in total phage the mutant frequency is $2.6 \pm 0.4 \%$.

The dot plots shown in Fig. 2 indicate the data from most of the DNAs are well-behaved. However, the first three transfections of each cell type with DNA carrying ethylguanine gave highly variable results. To examine this further two more transfections were carried out with AB1157 and four more with C600, using different batches of spheroplasts from each cell line. As shown in Fig. 2 there are no outliers; the data spreads evenly over a wide range. The reasons for this scatter in the EtG data are unknown.

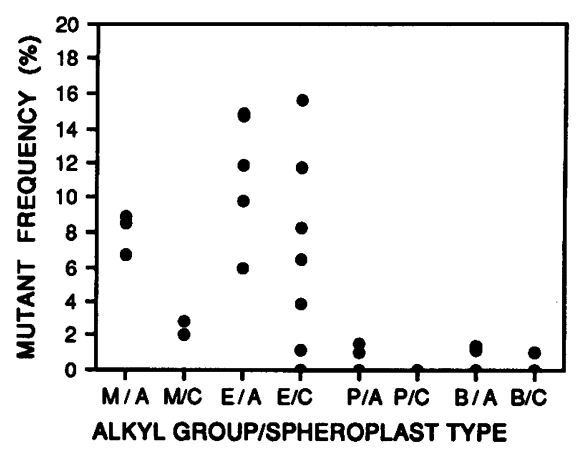

Figure 2. Variability of mutant frequencies from duplicate transfections. Alkyl groups: $\mathrm{M}=$ methyl, $\mathrm{E}=$ ethyl, $\mathrm{P}=$ propyl, $\mathrm{B}=$ butyl; spheroplast type: $\mathrm{A}=E$. coli $\mathrm{AB} 1157, \mathrm{C}=E$. coli $\mathrm{C} 600$

Table 1. Comparison of mutant frequencies introduced by transfection of $E$. coli $\mathrm{AB} 1157$ and $\mathrm{C} 600$ with $\phi X$ form $\mathrm{I}^{\prime}$ DNA carrying an $\mathrm{O}^{6}$-alkylguanine in the third codon of gene $G$.

\begin{tabular}{|c|c|c|c|c|c|c|c|c|}
\hline \multirow[b]{2}{*}{ DNA } & \multicolumn{4}{|c|}{ AB1157 } & \multicolumn{4}{|c|}{ C600 } \\
\hline & Mean & $S^{a}{ }^{a}$ & $\mathrm{~T}^{\mathrm{b}}$ & $\mathbf{P}^{c}$ & Mean & SD & $T$ & $\mathbf{P}$ \\
\hline C:MeG & 8.1 & 1.2 & 3. & 562 & 2.6 & 0.4 & 3 & 922 \\
\hline C:EtG & 11.4 & 3.7 & 5 & 1,307 & 5.9 & 5.7 & 7 & 1,963 \\
\hline C:PrG & 0.9 & 0.8 & 3 & 669 & 0 & - & 3 & 1,427 \\
\hline C:BuG & 0.9 & 0.8 & 3 & 771 & 0.3 & 0.6 & 3 & 834 \\
\hline
\end{tabular}

${ }^{\text {aStandard deviation }}$

${ }^{b}$ Number of transfections

${ }^{\mathrm{c}}$ Number 


\section{DISCUSSION}

These data show four things: (a) Within a given cell line, the mutant frequencies fall into two groups: $\mathrm{MeG}$ and $\mathrm{EtG}$, which are high; PrG and BuG, which are low. Based on a modified 't-test' the difference between these two groups is statistically significant at a confidence level $>99 \%$. Although the mean mutant frequencies for $\mathrm{EtG}$ are higher that those for $\mathrm{MeG}$ in both cell lines, the null hypothesis $\left(\mathrm{H}_{\mathrm{o}}: \mu_{\mathrm{MeG}}=\mu_{\mathrm{EtG}}\right)$ cannot be rejected at the $95 \%$ confidence level. This uncertainty is a reflection of the large variation in the EtG data. It should be noted that the decreasing order of mutant frequencies, Et $>\mathrm{Me}>$ $\mathrm{Pr}=\mathrm{Bu}$, found in $E$. coli $\mathrm{AB} 1157$ is similar to that found by classical mutagenesis in the same cells $(13,14)$. (b) The difference between the frequencies obtained with MeG-DNA in AB1157 and C600 is statistically significant at the $99 \%$ confidence level. There is also a considerable difference in the transfection means for Et-DNA in AB1157 and C600; however, because of the variation in the $\mathrm{EtG}$ data the null hypothesis, $\mathrm{H}_{\mathrm{o}}: \mu_{\mathrm{AB} 1157}=$ $\mu_{\mathrm{C} 600}$, cannot be rejected for this alkyl group. (c) PrG-DNA in C600 is the only case where no mutants were detected among the 1,427 plaques from five transfections that were examined. (d) A single $\mathrm{O}^{6}$-alkylguanine residue can produce a site-specific transition at a measurable frequency in cells with normal DNA repair systems; the widely held view to the contrary is no longer tenable in spite of negative results with $\mathrm{MeG}$ reported by other laboratories $(2,3,4)$.

The results with $\mathrm{MeG}$ and $\mathrm{BuG}$ in $E$. coli $\mathrm{AB} 1157$ agree with those reported earlier using the same experimental system but a different codon and phenotypic screen (1). However, the results with MeG in C600 do not agree with those of Bhanot and Ray (3); I found mutants without difficulty; they found none. There are a number of important differences in the experimental protocols used by the 2 laboratories, but the most important probably lie in the assays.

The mutant frequencies reported here represent the number of mutants present in samples drawn from the total phage population produced by artificial lysis of transfected spheroplasts. In this assay plaques are generated by amplification of individual phage particles in the lysate, and in general the plaques are homogeneous (unpublished data). The "infective center'" assay used by Bhanot and Ray does not measure the mutant frequency; it measures the number of plaques, each derived from a single transfected cell, that contain (arbitrarily) at least $10 \%$ mutant phage. Since the average mutant frequency for MeG in C600 is $3 \%$, many transfected cells will produce less than $10 \%$ mutants and fail to score in Bhanot and Ray's assay. Furthermore, the infective center assay, but not the 'total phage' assay, relies on amplification in situ of phage released by the transfected cell. Bhanot and Ray use $E$. coli $\mathrm{HF} 4740 \mathrm{Su}^{-}$recA/pBR $\phi X G$ for the amplification. These cells, which carry a functional copy of $\phi \mathrm{X}$ gene $\mathrm{G}$ on a plasmid, rescue the mutant by complementation. However, in this host, two factors, the amount of gene $G$ protein synthesized by the plasmid and a polar effect exerted by the Gam3 mutation on gene $\mathrm{H}$ expression, influence the efficiency of mutant rescue. Knowledge of the burst sizes of $w t$ and mutant phage on the host employed to amplify the phage is critical because the growth advantage for $w t$ phage over an amber mutant such as Gam3 can be as much as 20:1 (15). Bhanot and Ray do not give burst size data for $\mathrm{HF} 4740 \mathrm{Su}^{-} \mathrm{recA} / \mathrm{pBR} \phi \mathrm{XG}$ so the magnitude of the selective advantage is not known. However, even if the high copy number plasmid produces excess gene $G$ protein, the polar effect still favors wt phage during the amplification process; probably by a factor of at least $3(15,16)$. From this it follows that a transfected cell producing $3 \%$ mutants would produce a plaque $(\approx 107$ phage) with only $0.3 \%$ mutants in the infective center assay, and this would fail to score as a mutant plaque. In fact an individual transfected cell would have to produce at least $65 \%$ mutants before it would show up as a plaque containing $10 \%$ mutants. Thus, the infective center assay underestimates the true mutation frequency; this is particularly serious at low frequencies as is the case with $\mathrm{MeG}$ in $E$. coli C600.

It should be noted in passing that the mutant frequency is not the same as the mutation frequency. In the context of the experiments reported here the mutation frequency is the mispairing frequency per replication; this number, which is not easily accessible in experiments of this kind, is greater than the measured mutant frequency, which is influenced not only by the mispairing frequency but also the replication strand bias and DNA repair (for a discussion see ref. (17).

Our data with MeG cannot be compared directly with that reported by 2 other laboratories because their experiments were done with very different experimental systems $(2,4)$. However, it should be noted Hill-Perkins and coworkers used an infective center assay that may be subject to selective pressures favoring wt phage; Loechler et al. used a total phage assay but their experiments were done with a mixture of single stranded DNA's rather than purified form $I^{\prime}$ as I used.

Negative results not withstanding, it is clear that cells with normal repair systems can produce mutants at a measurable frequency and that the mutant frequency depends upon the nature of the alkyl group and the cell type. The mutant frequencies reported here represent a baseline against which changes produced by altering the repair status of the cell can be measured. Some initial results have been published $(1,17)$ and further studies of this kind are in progress.

\section{ACKNOWLEDGEMENTS}

I wish to thank Campbell for expert technical assistance. The work was supported by a grant from the Medical Research Council of Canada and by the John Stanton Memorial Fund.

\section{REFERENCES}

1. Chambers, R.W., Sledziewska-Gojska, E., Hirani-Hojatti, S. and BorowyBorowski, H. (1985) Proc. Natl. Acad.Sci. U.S.A, 82, 7173-7177.

2. Loechler, E.L., Green, C.L. and Essigmann, J.M. (1984) Proc. Natl. Acad. Sci. U.S.A., 81, 6271-6275.

3. Bhanot, O.S. and Ray, A. (1986) Proc. Natl. Acac. Sci. U.S.A., 83, $7348-7352$.

4. Hill-Perkins, M., Jones, M.D. and Karran, P. (1986) Mut. Res., 162, $153-163$.

5. Bhanot, O.S., Kahn, A. and Chambers, R.W. (1979) J. Biol. Chem., 254, $12684-12693$.

6. Chambers, R.W. (1989) Mutation Research, 210, 207-209.

7. Borowy-Borowski, H. and Chambers, R.W. (1987) Biochemistry, 26, 2465-2471.

8. Geschwinder, H.H. and Hofschneider, P.H. (1969) Biochim. Biophys. Acta 190, 454-459.

9. Malling, H.V., Fater, M.C., Burkhart, J.G., Hardies, S.C., Hutchison, C.A., III and Edgell, M.H. (1987) Gene Anal. Techn, 4, 23-26.

10. Guthrie, G.D. and Sinsheimer, R.L. (1963) Biochim. Biophys. Acta, 72, 290-297. 
2488 Nucleic Acids Research, Vol. 19, No. 9

11. Miller, J.H. (1972), Book Experiments in Molecular Genetics. Cold Spring Harbor Laboratory, New York, pp. Pages.

12. Cochran, W.G. (1964) Biometrics, 20, 191-195.

13. Schendel, P.F. and Robins, P. (1978) Proc. Natl. Acad. Sci. U.S.A., 75, 6017-6020.

14. Todd, M.L. and Schendel, P.F. (1983) J. Bact., 156, 6-12.

15. Humayun, M.Z. and Chambers, R.W. (1978) In Dressler, D. and Ray, D.S. (ed.), The Single-Stranded DNA Phages. Cold Spring Harbor Laboratory, New York, Vol. pp. 477-481.

16. Chambers, R.W., Kucan, I. and Kucan, Z. (1982) Nucleic Acids Res., 10, 6465-6473.

17. Chambers, R.W., Sledziewska-Gojska, E. and Hojatti-Hirani, S. (1988) Mol. Gen. Genet., 213, 325-331. 